\title{
Escaping from cycles through a glass transition
}

\author{
Sebastian Risau-Gusman* \\ Instituto de Física, Universidade Federal do Rio Grande do Sul, 91501-970 Porto Alegre, Rio Grande do Sul, Brazil
}

\author{
Alexandre S. Martinez $z^{\dagger}$ and Osame Kinouchi ${ }^{\ddagger}$ \\ Faculdade de Filosofia, Ciências e Letras de Ribeirão Preto, Universidade de São Paulo, Avenida Bandeirantes 3900 , \\ 14040-901 Ribeirão Preto, São Paulo, Brazil
}

(Received 20 December 2002; revised manuscript received 14 March 2003; published 11 July 2003)

\begin{abstract}
A random walk is performed over a disordered media composed of $N$ sites random and uniformly distributed inside a $d$-dimensional hypercube. The walker cannot remain in the same site and hops to one of its $n$ neighboring sites with a transition probability that depends on the distance $D$ between sites according to a cost function $E(D)$. The stochasticity level is parametrized by a formal temperature $T$. In the case $T=0$, the walk is deterministic and ergodicity is broken: the phase space is divided in a $O(N)$ number of attractor basins of two-cycles that trap the walker. For $d=1$, analytic results indicate the existence of a glass transition at $T_{1}$ $=1 / 2$ as $N \rightarrow \infty$. Below $T_{1}$, the average trapping time in two-cycles diverges and an out-of-equilibrium behavior appears. Similar glass transitions occur in higher dimensions when the right cost function is chosen. We also present some results for the statistics of distances for Poisson spatial point processes.
\end{abstract}

DOI: 10.1103/PhysRevE.68.016104

PACS number(s): 02.50.- r, 05.40.Fb, 05.90.+m, 87.15.Aa

\section{INTRODUCTION}

Random walks in random media constitute an interesting mathematical problem with several physical applications $[1,2]$. The study of deterministic walks in random media is also a fascinating topic but presents difficulties (nonergodicity, for instance) common to the area of dynamical systems. The statistics of such deterministic walks, which present transient and cycling parts, have been much less investigated [3-7] up to date. Here we investigate the transition from deterministic to random walks in a disordered medium. We find that escaping from a cycle dominated phase is done through a glass transition in the "weak ergodicity breaking" scenario present in Limoge-Bocquet [8] and Bouchaud's trap models [9-14].

Any spin lattice model with deterministic parallel update may be viewed as performing a deterministic walk in its phase space. Examples are the Little model [15,16] (a "Hopfield neural network" with parallel dynamics), asymmetric neural networks with parallel update [17], Kauffmann Boolean networks [18], etc. Since such systems have a huge and complex phase space, it is interesting to compare their behavior to other simpler dynamical systems with quenched disorder. This is the rationale for studying toy models as the random map [19] that shares some generic statistical and dynamical properties with those more complex systems.

A phase composed only by two-cycles (i.e., cycles involving only two states) occurs in the neural and Kauffman networks cited above. However, such two-cycle phase is not present in the random map model. Recently, we introduced a simple dynamical system with quenched disorder that pre-

\footnotetext{
*Electronic address: srisau@if.ufrgs.br

†Electronic address: amartinez@ffclrp.usp.br; URL: http:// www.fisicamedica.com.br/martinez/

${ }^{\ddagger}$ Electronic address: osame@dfm.ffclrp.usp.br
}

sents such a phase: the tourist walk [5-7]. It is a deterministic walk over a set of $N$ points. The points may be sites in a $d$-dimensional Euclidean space $[5,7]$ or nodes in a graph with ordered neighborhood (for example, words in a thesaurus [7]). The tourist rule is that at each time step, the walker must go to the nearest site not visited in the past $\tau$ steps (a self-avoiding window). We have found that, for any $\tau$ window, the phase space is divided in a $O(N)$ number of basins of $p$-cycles attractors. In particular, when $\tau=0$, all attractors have period $p=2$. For $\tau=1$, in the $d \rightarrow \infty$ limit, the tourist walk presents a power-law distribution of cycle periods of the form $P(p) \propto p^{-1}$, with a cutoff which scales with $\sqrt{N}$. This is similar to the distribution present in the random map model [19].

The above lattice systems also have been studied with stochastic dynamics. We expect that above a certain temperature, the dynamics is no longer dominated by the cycles. It is not clear how to introduce a stochastic dynamics in the random map but we found that it is easy (and even natural) to introduce a formal temperature $T$ in the tourist walk such that the deterministic limit is recovered when $T=0$. The main question we address here is how ergodicity could be recovered from such deterministic dynamical systems with quenched disorder. The introduction of dynamical stochasticity is necessary to break the cycles, but a low level of stochasticity may not be sufficient for full ergodicity recovery. Indeed, we have found a glasslike transition at a finite stochasticity level (temperature) $T_{c}$. We expect that a similar behavior is present in, for example, stochastic versions of Kauffman networks.

Here we consider a stochastic tourist walk where the walker, at site $i$, is allowed to jump to its first $n$ neighbors with a transition probability proportional to $\exp \left[-E\left(D_{i j}\right) / T\right]$. The stochasticity level is parametrized by $T$ (a formal temperature) and the cost function $E\left(D_{i j}\right)$ is a monotone increasing function of the distance between the sites. When the formal temperature $T$ is zero, one has a simple tourist walk 
without self-avoiding window $(\tau=0)$ : the walker always goes to the nearest neighboring site, eventually being trapped in a two-cycle when a pair of reciprocally nearest neighbors is found. For $T>0$, the two-cycles are no longer stable, being characterized by a distribution of trapping times $P\left(t_{r}\right)$. However, for low values of $T$, the walker is in an out-ofequilibrium regime, presenting a divergent average trapping time when $N \rightarrow \infty$ (weak ergodicity breaking scenario [8-14]). Increasing $T$, one is able to study the ergodicity recovery transition where the average trapping time becomes finite. For one-dimensional systems, this occurs at $T_{1}=1 / 2$ for all values of the connectivity $n$.

The paper is organized as follows. In Sec. II, we define the model and set the notation. In Sec. III, we show that the network structure, where hops occur, forms a directed graph. This graph presents interesting sets of points that we call sinks and sources. We present analytic results on the density of sinks of a given size. In Sec. IV, we obtain some joint distributions of distances for the Poisson spatial point process. These results are used in the calculation of bounds for the average trapping time in two-cycles. In Sec. V, we show that in one-dimensional systems there is a glass transition at $T_{1}=1 / 2$. Below $T_{1}$, the average trapping time diverges and the system falls into an out-of-equilibrium state. A discussion on the existence of such glass transitions in higher dimensions is presented. Exploratory behavior with a power-law hopping process is discussed via the introduction of a proper cost function. Our final remarks and conclusions are offered in Sec. VII.

\section{DESCRIPTION OF THE MODEL}

The model is defined by an underlying disordered lattice composed of $N$ sites, whose coordinates $x_{i}^{(k)}(i=1, \ldots, N$; $k=1, \ldots, d)$ are distributed uniformly in the $d$-dimensional unitary volume $[0,1]^{d}$. From the sites coordinates, one constructs the Euclidean distance matrix:

$$
D_{i j} \equiv N^{1 / d}\left\{\sum_{k=1}^{d}\left[x_{i}^{(k)}-x_{j}^{(k)}\right]^{2}\right\}^{1 / 2},
$$

where the factor $N^{1 / d}$ has been introduced to simulate a constant density of points, when the thermodynamical limit $N$ $\rightarrow \infty$ is taken. In this limit, the point distribution is equivalent to that of a Poisson process in $\mathbb{R}^{d}$, using the geometrical distance.

Now consider a hopping process between sites. The transition probability from site $i$ to site $j$ is defined as

$$
W_{i \rightarrow j}(\beta)=\frac{e^{-\beta E\left(D_{i j}\right)}}{Z_{i}},
$$

where the normalization factor is

$$
Z_{i}(\beta)=\sum_{j \in \mathrm{NN}} e^{-\beta E\left(D_{i j}\right)}
$$

with the sum running over the $n$ nearest neighbors $(\mathrm{NN})$ of point $i$. The cost function $E\left(D_{i j}\right)$ depends only on the distances $D_{i j}$, and is a monotone increasing function.

Several scenarios can be envisaged. If $E\left(D_{i j}\right)=D_{i j}$, then the transition probability decays exponentially with the distance. If $E\left(D_{i j}\right)=D_{i j}^{2}$, a Gaussian decay is obtained for $W_{i \rightarrow j}$. If $E\left(D_{i j}\right)=\ln D_{i j}$, then $W_{i \rightarrow j} \propto D_{i j}^{-\beta}$ decays as a power law of the distance.

Notice that the transition probabilities are not symmetric $\left(W_{i \rightarrow j} \neq W_{j \rightarrow i}\right)$ because the normalization factors are different $\left(Z_{i} \neq Z_{j}\right)$ : the neighbors of $i$ are not the same as the neighbors of $j$. Furthermore, the walker is not allowed to remain at the same site, i.e., $W_{i \rightarrow i}=0$ (which is equivalent to setting $D_{i i}=\infty$ in the distance matrix).

The stochastic parameter or "temperature" $T=1 / \beta$ regulates the distance dependent bias of the model. When $\beta$ $\rightarrow 0$, all the transition probabilities are equal and one obtains unbiased hopping inside a disordered lattice. When $\beta \rightarrow \infty$, in Eq. (3) the term corresponding to the first-nearest neighbor dominates, yielding either $W_{i \rightarrow j}=1$, if the site $j$ is the nearest neighbor of site $i$, or $W_{i \rightarrow j}=0$, otherwise. The tourist goes imperatively to the nearest site, relative to the actual position, thus performing a deterministic walk [5] without a self-avoiding window (that is, with $\tau=0$ ).

Observe that the model has not been defined in a regular lattice because in that case the number of nearest neighbors is degenerated (greater than one) and determinism cannot be recovered. The prohibition for the walker to remain at the same site is needed to fulfill the tourist walk rule.

When $n=N-1$, any site is accessible from any other site. However, if $n$ is small, the underlying network is a diluted directed graph. Such graphs have a complicated topology: some sites may not be accessible from other sites and the graph may be disconnected. Thus, we will first study the topology induced in this graph by a finite degree $n$ of outgoing links. Afterwards, we present the dynamical ("glass") transition that occurs in the walks performed on this graph, when $T$ is sufficiently low.

\section{TOPOLOGY OF THE UNDERLYING NETWORK}

We have assumed that transitions occur only to the first $n$ nearest neighbors of each site $i$. Viewing each transition term $W_{i \rightarrow j}$ as the weight of the link between sites $i$ and $j$, we obtain a directed network or graph. The degree distribution of outgoing links is a $\delta$ function, $P\left(k_{\text {out }}\right)=\delta_{k, n}$, and the distribution of ingoing links $P\left(k_{i n}\right)$ is binomial. Notice, however, that this is not a random graph, as it is restricted to a $d$-dimensional Euclidean space, and thus it cannot be fully characterized by using only the distribution of ingoing and outgoing links. This graph is the underlying network where deterministic tourist walks occur (if we set $n=\tau+1$ ), so it has been called a tourist graph.

\section{A. Sinks and sources}

For all finite values of $n$, topological constraints induce special sets of points. For example, using $n=2$ and $d=2$, consider a set of three points such that each point has the 


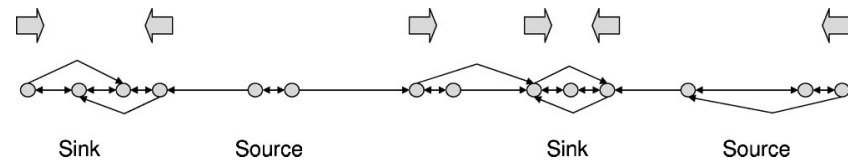

FIG. 1. Sinks and sources in one dimension. Large arrows represent walls.

other two points as nearest neighbors. All the outgoing links of such points remain inside this set of three points, forming a triangle. If this triangle does not receive any ingoing link from outside points, this triangle is an isolated cluster. If it receives some ingoing links, it acts as an absolute trap: the cluster may be attained from outside, but there is no possibility to escape from it, since there are no transition terms $W_{i \rightarrow j}$ to any site $j$ outside the triangle. Such sets, which do not present outgoing links, we call sinks. When these sinks are minimal (i.e., there are no smaller sinks inside them), they correspond to what are called irreducible closed sets in the terminology of Markov processes [20]. Sinks are composed of persistent states and the rest of the graph is composed of transient ones [20]. Notice that, since there are always $n$ outgoing links from each point, a single site cannot be a sink. Sinks have a minimal size of $n+1$ points.

Consider also a set of points (or even a single point) that present outgoing links to points outside the given set, but with no ingoing links from exterior points. Such sets are called sources. A walk may be started inside a source, but if the walker exits this set, she cannot return to it any longer.

Sinks and sources are easily visualized in dimension $d$ $=1$ (see Fig. 1) because we can define special points ("walls"). A site $w$ is a (semipermeable) wall whenever all of its $n$ outgoing links point along the same direction. However, $w$ may have ingoing links from the opposite direction, that is, the walls behave as semipermeable membranes: they allow the flux of walkers from one side to another but not the other way around. Thus the walls are characterized by the directions in which the walker flux is allowed. This is indicated in Fig. 1 by arrows and, as a shorthand, we say that the wall "points" to the direction indicated by the large arrows.

In $d=1$ systems, two consecutive walls define different regions on the disordered lattice depending on their orientation. If the two walls point to the center of a region (inwards) $(|\Rightarrow \Leftarrow|)$, the set of points comprising all the points between the walls is a minimal sink. Once a walker attains a sink, there is no escape: the only permissible movement is to wander stochastically inside it. If the two consecutive walls point outwards a region $(\Leftarrow \| \Rightarrow)$, this region is a source. Once a walker escapes from such a region, it cannot return.

Going to a higher level of description, our graph can be characterized by the sets discussed in Refs. [21] and [22]. For $d>1$ and large enough values of $n$, the graph has a giant weakly connected component made of points connected by links (without considering their direction). Inside this set, there is a giant strongly connected component (GSCC), where every point is accessible from every other point of the set through a directed path. This is the set where, most probably, a random walker roams until she eventually falls into a sink. By definition [22], the GSCC does not contain either sinks or sources. The (disjoint) set of sources whose outgo- ing links lead to the GSCC is the in-component (IN), i.e., the set of points from where the GSCC is reachable but cannot be reached from it. If the walker is initially placed in the IN, she will leave this set for the GSCC in a relatively small amount of time. The disjoint set of sinks that can be reached from the GSCC is called the out-component (OUT). This is the set where almost every random walker will end up. These are the greatest sets in our graph, but other smaller sets are possible, for example, the set of all the sources that are not connected to the GSCC, but to other sinks; or the set of sinks that cannot be reached from any other point in the graph.

In $d=1$, due to the very special topology of $\mathbb{R}$, things are different. Sinks disconnect the graph (nothing passes from one side to the other side of a sink) and a GSCC cannot be formed.

Notice that the presence of sources and sinks occurs even in the high temperature limit $(T \rightarrow \infty)$. It is a topological property, not a dynamical one, emerging from the fact that each site has only $n$ outgoing links. The glass transition discussed in Sec. V has nothing to do with such topological constraints. Simulations show that it is present even in the fully connected case $n=N-1$. Sinks are an undesirable (although inevitable) factor that complicates the study of the glass transition in our random walk. On the other hand, finite connectivity allows us to extract some analytical results, which is not the case for $n \rightarrow \infty$.

\section{B. Density of sinks}

Next we calculate the density of sinks in one dimension and ask for the conditions which enable the emergence of a single macroscopic connected region where any point is reachable from any other.

In $d=1$, a sink is formed by two inward walls where each wall site has $n$ outgoing links. Thus, the smallest sink comprises $n+1$ points. It is possible to calculate explicitly the density (also called concentration) $C_{m}(n)$ of sinks with sizes $n+m$ for $m$ small.

Consider a set of $n+3$ consecutive points, relabeled as $i$ $=1, \ldots, n+3$ and with coordinates $x_{i}$. Let $X_{i}=x_{i+1}-x_{i}$ be the distance separating two consecutive points. We first calculate the probability that point $i=2$ is a wall pointing to the right and that, simultaneously, point $i=n+2$ is a wall pointing to the left with no wall between these two walls. If $X_{1}$ is greater than the total distance between points $i=2$ and $i$ $=n+2$, then point $i=2$ is a left wall pointing to the right. Similarly, if $X_{n+2}$ is greater than the total distance between points $i=2$ and $i=n+2$, then point $i=n+2$ is a right wall pointing to the left. Since points are randomly spaced along the line (Poisson process), the probability of having a distance $X_{i}$ is $P\left(X_{i}\right)=e^{-X_{i}}$. Thus, the concentration of sinks having $n+1$ points is

$$
\begin{aligned}
C_{1}(n)= & \int \prod_{i=1}^{n+2}\left(d X_{i} e^{-X_{i}}\right) \Theta\left(X_{1}-\sum_{i=2}^{n+1} X_{i}\right) \\
& \times \Theta\left(X_{n+2}-\sum_{i=2}^{n+1} X_{i}\right)=\frac{1}{3^{n}} .
\end{aligned}
$$


TABLE I. Expression of function $p_{d}(x)$ for some values of the dimension $d$ [see Eq. (8)]. This generalizes Table II of Ref. [7]. For the last column, see Eq. (21).

\begin{tabular}{lccc}
\hline \hline$d$ & $p_{d}(x)$ & $p_{d}(1)$ & $\partial V_{\cup}\left(D_{1}, D_{0}\right) / \partial D_{1}$ \\
\hline 1 & $\frac{x}{2}$ & $\frac{1}{2}$ & 2 \\
2 & $\frac{2}{\pi}\left[\frac{x}{2} \sqrt{1-\left(\frac{x}{2}\right)^{2}+\arctan \sqrt{\left.\frac{(x / 2)^{2}}{1-(x / 2)^{2}}\right]}}\right.$ & $\frac{3^{3 / 2}+2 \pi}{6 \pi}$ & $2 \pi D_{1}+4 D_{1} \arctan \sqrt{\frac{\left[D_{1} /\left(2 D_{0}\right)\right]^{2}}{1-\left[D_{1} /\left(2 D_{0}\right)\right]^{2}}}$ \\
3 & $\frac{3}{2}\left[\frac{x}{2}-\frac{1}{3}\left(\frac{x}{2}\right)^{3}\right]$ & $\frac{11}{16}$ & $2 \pi D_{1}\left[2 D_{1}+D_{0}\right]$ \\
$\vdots$ & $\vdots$ & $\vdots$ & $\vdots$ \\
\hline \hline
\end{tabular}

Similar (but more cumbersome) calculations give the concentration for the next two sink sizes $(n+2$ and $n+3)$. For $n \geqslant 2$,

$$
\begin{gathered}
C_{2}(n)=\frac{1}{4}\left(\frac{1}{3^{n-1}}-\frac{1}{5^{n-1}}\right), \\
C_{3}(n)=\frac{1}{16}\left(\frac{1}{3^{n-2}}-\frac{1}{5^{n-2}}+\frac{1}{9} \frac{1}{7^{n-2}}+\frac{1}{12} \frac{1}{9^{n-2}}\right) .
\end{gathered}
$$

For large values of $n, \quad C_{2}(n) / C_{1}(n) \rightarrow 3 / 4 \quad$ and $C_{3}(n) / C_{2}(n) \rightarrow 3 / 4$. One may conjecture that, asymptotically, this will hold true for all size concentrations. In other words, $C_{m+1}(n) / C_{m}(n) \rightarrow 3 / 4$ for all values of $m$. If this conjecture is valid, the fraction of points that belong to sinks is, asymptotically, $\quad F(n)=\sum_{m=1}^{\infty}(m+n) C_{m}(n)=(4 n+20) / 3^{n}$ and the total concentration of sinks is $C(n)=\sum_{m=1}^{\infty} C_{m}(n)$ $=4 / 3^{n}$. This means that for large values of $n$, the graph is effectively disconnected and the clusters have, on average, $[1-F(n)] / C(n)=\left(3^{n}-4 n-20\right) / 4$ points.

This result can be used to estimate the minimum value of $n$ for which there exists a single cluster of size $O(N)$. If one imposes $N C(n)=1$, one gets $n_{\text {min }}=\ln (4 N) / \ln 3$. This implies that it is not necessary to take $n=N-1$ to have a single macroscopic cluster: it can be obtained with very high probability for low values $[O(\ln N)]$ of $n$.

It is easy to see that for higher dimensions and finite $n$, there will also be a finite fraction of sinks and sources, in the thermodynamical limit $N \rightarrow \infty$. However, numerical evidence presented in Sec. V B seems to imply that the glass transition studied in Sec. V occurs in the GSCC, that is, outside the sinks and sources.

\section{COUPLE STATISTICS}

In this section, we obtain the density of couples (which form two-cycles traps) in $d$ dimensions and we derive probability density functions for distances inside couples and between couples and their neighbors.

\section{A. Density of couples}

With $T=0$, starting the walk at a given site, after a fast transient [23], the walker eventually reaches two "reflexive nearest neighbors" (or "couple," for short) [24]. A couple $a \leftrightarrow b$ is formed when site $a$ is the nearest neighbor of site $b$ and $b$ is the nearest neighbor of $a$. Once a couple is attained, the walker is trapped in a two-cycle attractor $(a-b-a-b$ $-\cdots)$. As shown in Ref. [7], for $d=1$, the expected number of couples is $M(1)=N / 3$, and for general values of $d$ one has the couple density:

$$
\frac{M(d)}{N}=\frac{1}{2} P_{d}(a \leftrightarrow b)=\frac{1}{2\left[1+p_{d}(1)\right]},
$$

where $P_{d}(a \leftrightarrow b)$ is the probability that a point belongs to a couple and

$$
p_{d}(x)=I_{(x / 2)^{2}}\left(\frac{1}{2}, \frac{d+1}{2}\right),
$$

with the definitions [25]

$$
\begin{gathered}
I_{z}(a, b)=\frac{1}{B(a, b)} \int_{0}^{z} d t t^{a-1}(1-t)^{b-1}, \\
B(a, b)=\int_{0}^{1} d t t^{a-1}(1-t)^{b-1}=\frac{\Gamma(a) \Gamma(b)}{\Gamma(a+b)}, \\
\Gamma(z)=\int_{0}^{\infty} d t t^{z-1} e^{-t},
\end{gathered}
$$

where $I_{z}(a, b)$ is the incomplete $\beta$ function, $B(a, b)$ is the beta function, and $\Gamma(z)$ is the gamma function. The function $p_{d}(x)$ is given in Table I for some values of $d$, and its geometrical meaning is presented below (Sec. IV B).

The couples trap permanently the walker only at $T=0$. Notice that, in principle, there is no relationship between couples and sinks (for example, couples may be sources). However, the special case $n=1$ (a single outgoing link for each point) is equivalent to a $T=0$ scenario. In this case, sinks and couples are identical. From Eq. (4), $C_{1}(1)=1 / 3$ gives the concentration of couples, which is compatible with Eq. (7), for $d=1$. 


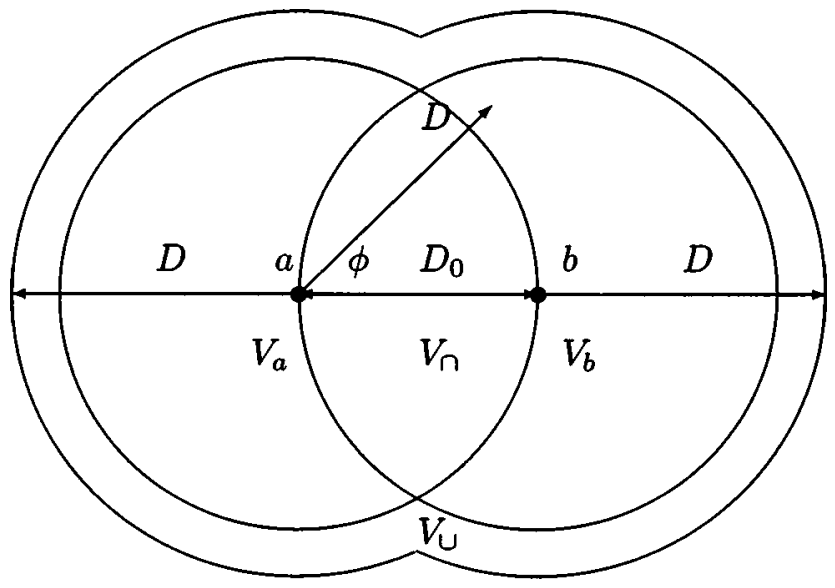

FIG. 2. Union of two hyperspheres of radius $D$ centered at sites $a$ and $b$, which are a distance $D_{0}$ apart. There are no other sites inside the inner hyperspheres of radius $D_{0}$.

As we are using a spatial Poisson process, the probability of a point being located in a given volume is proportional to the volume itself and there is no correlation between points. Next we present some analytical results on the distance statistics of point processes in $\mathbb{R}^{d}$ that could be of general interest (for example, in studies of the travelling salesman problem [26]).

\section{B. Couple distance distribution function}

First we calculate the probability density function (PDF) for the distances between the points of a couple. Following Cox [24], without loss of generality, we consider a unitary intensity Poisson process in $d$ dimensions. For such a process, the probability of having no points inside a hypersphere of volume $V$ and radius $D$ is $e^{-V}$. Then, the PDF of finding the nearest neighbor of a particular site $a$ at a distance $D$ is

$$
P_{n n}(D)=\frac{d V(D)}{d D} e^{-V(D)}=A_{d} d D^{d-1} e^{-V(D)},
$$

with

$$
\begin{gathered}
V(D)=A_{d} D^{d}, \\
A_{d}=\frac{\pi^{d / 2}}{\Gamma(d / 2+1)} .
\end{gathered}
$$

Consider also the volume of the union of two $d$ spheres of radius $D$ centered at sites $a$ and $b$ separated by a distance $D_{0}$ (see Fig. 2):

$$
V_{\cup}\left(D, D_{0}\right)=2 V(D)-V_{\cap}\left(D, D_{0}\right)
$$

where $V_{\cap}\left(D, D_{0}\right)$ is the volume of the intersection of two $d$ spheres whose centers are separated by a distance $D_{0}$. Using the variable $x=\cos \phi$ (see Fig. 2), we get

$$
\begin{aligned}
V_{\cap}\left(D, D_{0}\right) & =\frac{2 V(D)}{B\left(\frac{1}{2}, \frac{d+1}{2}\right)} \int_{D_{0} / 2 D}^{1} d x\left(1-x^{2}\right)^{(d-1) / 2} \\
& =\frac{V(D)}{B\left(\frac{1}{2}, \frac{d+1}{2}\right)} \int_{\left(D_{0} / 2 D\right)^{2}}^{1} d t t^{-1 / 2}(1-t)^{(d-1) / 2} \\
& =V(D)\left[1-p_{d}\left(\frac{D_{0}}{D}\right)\right] .
\end{aligned}
$$

Notice that $p_{d}(x)=\left[V(D)-V_{\cap}\left(D, D_{0}\right)\right] / V(D)$ is the volume fraction occupied by the "hyper crescent." Thus, the total volume is

$$
V_{\cup}\left(D, D_{0}\right)=V(D)\left[1+p_{d}\left(\frac{D_{0}}{D}\right)\right] .
$$

The joint PDF that the points $a$ and $b$ form a couple and have a pair distance $D_{0}$ corresponds to the probability that no other points lie inside the volume $V_{\cup}\left(D_{0}, D_{0}\right)$ :

$$
\begin{aligned}
P_{d}\left(D_{0}, a \leftrightarrow b\right) & =\frac{d V\left(D_{0}\right)}{d D_{0}} e^{-V_{\cup}\left(D_{0}, D_{0}\right)} \\
& =A_{d} d D_{0}^{d-1} e^{-\left[1+p_{d}(1)\right] V\left(D_{0}\right)} .
\end{aligned}
$$

For example, in $d=1$ we have $P_{1}\left(D_{0}, a \leftrightarrow b\right)=2 e^{-3 D_{0}}$. Notice that $P_{d}\left(D_{0}, a \leftrightarrow b\right)$ is not normalized: integrating with respect to $D_{0}$ one recovers the couple density $P_{d}(a \leftrightarrow b)$ given by Eq. (7).

\section{Couple nearest neighbor distance distribution function}

Define $D_{j}$ as the distance of the $j$ th nearest neighbor (1 $\leqslant j \leqslant n$ ) to the couple $a \leftrightarrow b$. For instance, the distance of the first-nearest neighbor to the couple is $D_{1}=\min \left\{D_{a 1}, D_{b 1}\right\}$, that is, the minimal one between the distance from sites $a$ and $b$ to their second-nearest neighbors. Thus, the distance of the second-nearest neighbor to the couple will be the minimal one among the remaining distances $\left(\max \left\{D_{a 1}, D_{b 1}\right\}\right)$ and $\left\{D_{a 2}, D_{b 2}\right\}: D_{2}=\min \left\{\max \left\{D_{a 1}, D_{b 1}\right\}, D_{a 2}, D_{b 2}\right\}$.

To calculate the couple's joint PDF $P\left(D_{0}, D_{1}\right)$, one asks first for the probability that the couple nearest neighbor is at a distance $r$ larger than $D_{1}$. That is, one asks for the probability that two $d$ spheres of radius $D_{1}$, one centered at site $a$ and the other centered at site $b$, are empty, given the fact that the $d$ spheres of radius $D_{0}$ centered at the same sites are also empty. For a Poisson process, this probability is

$$
P_{d}\left(r>D_{1} \mid D_{0}\right)=\frac{e^{-V_{\cup}\left(D_{1}, D_{0}\right)}}{e^{-V_{\cup}\left(D_{0}, D_{0}\right)}} .
$$

Then, the probability density function that $r>D_{1}$ is

$$
\begin{aligned}
P_{d}\left(r>D_{1}, D_{0}\right) & =P_{d}\left(r>D_{1} \mid D_{0}\right) P_{d}\left(D_{0}, a \leftrightarrow b\right) \\
& =\frac{d V\left(D_{0}\right)}{d D_{0}} e^{-V_{\cup}\left(D_{1}, D_{0}\right)},
\end{aligned}
$$


where $P_{d}\left(D_{0}, a \leftrightarrow b\right)$ has been given by Eq. (17). Taking the derivative of the above equation with respect to $D_{1}$, one finally obtains the probability distribution function to have a couple $a \leftrightarrow b$ of size $D_{0}$ with the first neighbor to the couple at a distance $D_{1}$ :

$$
\begin{aligned}
P_{d}\left(D_{1}, D_{0}, a \leftrightarrow b\right) \\
=\exp \left[-V_{\cup}\left(D_{1}, D_{0}\right)\right] \frac{d V\left(D_{0}\right)}{d D_{0}} \frac{\partial V_{\cup}\left(D_{1}, D_{0}\right)}{\partial D_{1}} \\
\quad \times \Theta\left(D_{1}-D_{0}\right),
\end{aligned}
$$

where the Heaviside step function $\Theta\left(D_{1}-D_{0}\right)$ has been introduced to ensure that $D_{1}>D_{0}$ and

$$
\frac{\partial V_{\cup}\left(D_{1}, D_{0}\right)}{\partial D_{1}}=\frac{V_{\cup}\left(D_{1}, D_{0}\right) d}{D_{1}}+\frac{\partial p_{d}\left(D_{0} / D_{1}\right)}{\partial D_{1}} V\left(D_{1}\right),
$$

where

$$
\frac{\partial p_{d}\left(D_{0} / D_{1}\right)}{\partial D_{1}}=\frac{-D_{0}\left[1-\left(\frac{D_{0}}{2 D_{1}}\right)^{2}\right]^{(d-1) / 2}}{D_{1}^{2} B\left(\frac{1}{2}, \frac{d+1}{2}\right)}
$$

For the expression of $\partial V_{\cup}\left(D_{1}, D_{0}\right) / \partial D_{1}$ for some values of $d$, see Table I.

\section{Generalizing the joint distribution function}

Generalizing this procedure, the joint PDF of distances for the $k$ th nearest neighbors of the $a \leftrightarrow b$ couple is obtained:

$$
\begin{aligned}
P_{d}\left(D_{k}, D_{k-1}, \ldots, D_{0}, a \leftrightarrow b\right) \\
=\exp \left[-V_{\cup}\left(D_{k}, D_{0}\right)\right] \frac{d V\left(D_{0}\right)}{d D_{0}} \prod_{j=1}^{k}\left[\frac{\partial V_{\cup}\left(D_{j}, D_{0}\right)}{\partial D_{j}}\right. \\
\left.\quad \times \Theta\left(D_{j}-D_{j-1}\right)\right]
\end{aligned}
$$

Notice that the above result is not normalized (stressed by the label $a \leftrightarrow b)$. To normalize it, one must divide by $P_{d}(a \leftrightarrow b)=1 /\left[1+p_{d}(1)\right][$ see Eq. (7)]. For instance, for $d$ $=1$ one has

$$
P_{1}\left(D_{k}, D_{k-1}, \ldots, D_{0}\right)=2^{k} 3 e^{-\left(D_{0}+2 D_{k}\right)} \prod_{i=1}^{k} \Theta\left(D_{i}-D_{i-1}\right) \text {. }
$$

Notice that normalized PDF's do not carry the label $a \leftrightarrow b$.

\section{E. Stability distribution function}

Let us define the "stability" of an arbitrary pair of points $(a, b)$ (not necessarily reflexive neighbors) to be

$$
\Delta=D_{n n}-D_{a b},
$$

TABLE II. Probability $P(t)$ that a walker, initially placed at point $a$, makes its first exit from the couple at time $t$.

\begin{tabular}{cc}
\hline \hline Time step $(t)$ & $P(t)$ \\
\hline 0 & $q_{a}$ \\
1 & $p_{a} q_{b}$ \\
2 & $p_{a} p_{b} q_{a}$ \\
3 & $p_{a} p_{b} p_{a} q_{b}$ \\
4 & $\left(p_{a} p_{b}\right)^{2} q_{a}$ \\
5 & $\left(p_{a} p_{b}\right)^{2} p_{a} q_{b}$ \\
6 & $\left(p_{a} p_{b}\right)^{3} q_{a}$ \\
$\vdots$ & $\vdots$ \\
$t$ odd & $\left(p_{a} p_{b}\right)^{(t-1) / 2} p_{a} q_{b}$ \\
$t$ even & $\left(p_{a} p_{b}\right)^{t / 2} q_{a}$ \\
\hline \hline
\end{tabular}

where $D_{n n}=\min \left\{D_{a k}, D_{b k}\right\}$ is the distance to the nearest neighbor $k$ of $a$ or $b$ not including $a$ or $b$. Notice that if (and only if) the stability $\Delta$ is positive, then $a$ and $b$ are reflexive neighbors (a couple). This is an alternative way of defining couples and we may write $\Delta=D_{1}-D_{0}$. The couple stability $\mathrm{PDF}$ is

$$
P_{d}(\Delta)=\int_{0}^{\infty} d D_{0} d D_{1} P_{d}\left(D_{1}, D_{0}\right) \delta\left[\Delta-\left(D_{1}-D_{0}\right)\right] .
$$

For example, for $d=1$ this gives

$$
P_{1}(\Delta)=2 \exp (-2 \Delta) \Theta(\Delta) .
$$

\section{GLASS TRANSITION}

So far we have considered the topological aspects of the network where the walk is performed. Now we turn our attention to the dynamics of the hopping process. As mentioned before, for $T=0$ the walk is deterministic. After a transient, the walker falls into a two-cycle defined by a couple. However, for $T>0$ the couples are not absolute traps any longer, being characterized by a residence or trapping time, $t_{r}$. To calculate the average residence time let $a$ and $b$ be the sites of a couple, $D_{0}=D_{a b}$ their pair distance, and $D_{a j}\left(D_{b j}\right)$ the distance from $a(b)$ to its $(j+1)$ th nearest neighbor. Consider first a linear cost function $E(D)=D$ (other cost functions will be discussed later). The probabilities of transition from site $a$ to site $b$ and vice versa are

$$
\begin{aligned}
& p_{a} \equiv W_{a \rightarrow b}(\beta)=\frac{e^{-\beta D_{0}}}{Z_{a}(\beta)}, \quad Z_{a}(\beta)=\sum_{i=0}^{n-1} e^{-\beta D_{a i}}, \\
& p_{b} \equiv W_{b \rightarrow a}(\beta)=\frac{e^{-\beta D_{0}}}{Z_{b}(\beta)}, \quad Z_{b}(\beta)=\sum_{i=0}^{n-1} e^{-\beta D_{b i}} .
\end{aligned}
$$

Suppose that at $t=0$ the walker is at site $a$. The probability that the walker remains inside the couple for $t$ steps and then leaves it is (as schemed in Table II): $P_{e}(t)$ $=\left(p_{a} p_{b}\right)^{t / 2} q_{a}$, for even values of $t$ and $P_{o}(t)$ $=\left(p_{a} p_{b}\right)^{(t-1) / 2} p_{a} q_{b}$ for odd values of $t$, with $q_{a}=1-p_{a}$ and 
$q_{b}=1-p_{b}$. If at $t=0$ the walker is at site $b$, one simply has to exchange the indices $a$ and $b$ in $P_{o}(t)$ and $P_{e}(t)$. Since the travel may start either at site $a$ or site $b$, the probabilities that the walker remains in the cycle up to time $t$ are $P_{e}(t)$ $=\left(p_{a} p_{b}\right)^{t / 2}\left(q_{a}+q_{b}\right) / 2 \quad$ and $\quad P_{o}(t)=\left(p_{a} p_{b}\right)^{(t-1) / 2}\left(p_{a} q_{b}\right.$ $\left.+p_{b} q_{a}\right) / 2$, with $P(t)$ properly normalized: $\sum_{t=0}^{\infty} P(t)$ $=\sum_{k=0}^{\infty}\left[P_{e}(2 k)+P_{o}(2 k+1)\right]=1$.

The expected residence time for this couple is

$$
\begin{aligned}
t_{r} & =\sum_{t=0}^{\infty} P(t) t=\sum_{k=0}^{\infty}\left[P_{e}(2 k) 2 k+P_{o}(2 k+1)(2 k+1)\right] \\
& =\frac{p_{a} p_{b}+\left(p_{a}+p_{b}\right) / 2}{1-p_{a} p_{b}}
\end{aligned}
$$

The calculation of the average $\left\langle t_{r}\right\rangle$ (over all possible realizations of the points) is somewhat involved, for general values of $n$. The problem lies in the fact that the set of nearest neighbors to site $a$ and the corresponding one for site $b$ are neither uncorrelated (independent) nor completely correlated.

The consideration of all the possible correlations involves a number of integrals that increases exponentially with $n$. Nevertheless, it is possible to calculate bounds for $\left\langle t_{r}\right\rangle$ which reveal its characteristic diverging behavior. To obtain these bounds, we next present a simple approximation which is physically intuitive followed by improvements to this calculation.

\section{A. Symmetrical and asymmetrical approximations}

In the symmetrical approximation, for $n=2$, the transition probabilities corresponding to the two members of a couple

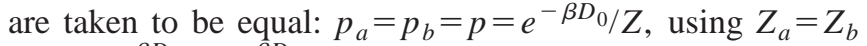
$=Z=e^{-\beta D_{0}}+e^{-\beta D_{1}}$, with $D_{1}=\min \left(D_{1 a}, D_{1 b}\right)$ (as defined in Sec. IV C). The residence time is then given by $t_{r s}=p /(1$ $-p)=e^{\beta \Delta}$, where $\Delta=D_{1}-D_{0}$ is the stability of the couple, defined by Eq. (25). This would be exact if the nearest neighbors to the couple were degenerated, that is, if there was one point at a distance $D_{1}$ from $a$ and a point at a distance $D_{1}$ from $b$.

In the general case, $t_{r s}$ is a lower bound to $t_{r}$. This stems from the very definition of $D_{1}$ : for one of the points of the couple, the distance to its second-nearest neighbor (SNN) is, in the general case, necessarily larger than $D_{1}$. Thus, given $D_{1}$, a couple whose members have their respective SNNs at the same distance, $D_{1}$, has the shortest possible residence time.

In the asymmetrical approximation, for the member of the couple (say, $b$ ) whose SNN is at a distance larger than $D_{1}$, the transition probability to the SNN is taken to be zero, that is, $p_{b}=1$. This leads to a residence time $t_{r a}=2 e^{\beta \Delta}+1 / 2$. This residence time clearly bounds the true residence time from above, because we are assuming that the walker cannot leave the couple from point $b$.

From these approximations, one obtains

$$
e^{\beta \Delta} \leqslant t_{r} \leqslant 2 e^{\beta \Delta}+\frac{1}{2} .
$$

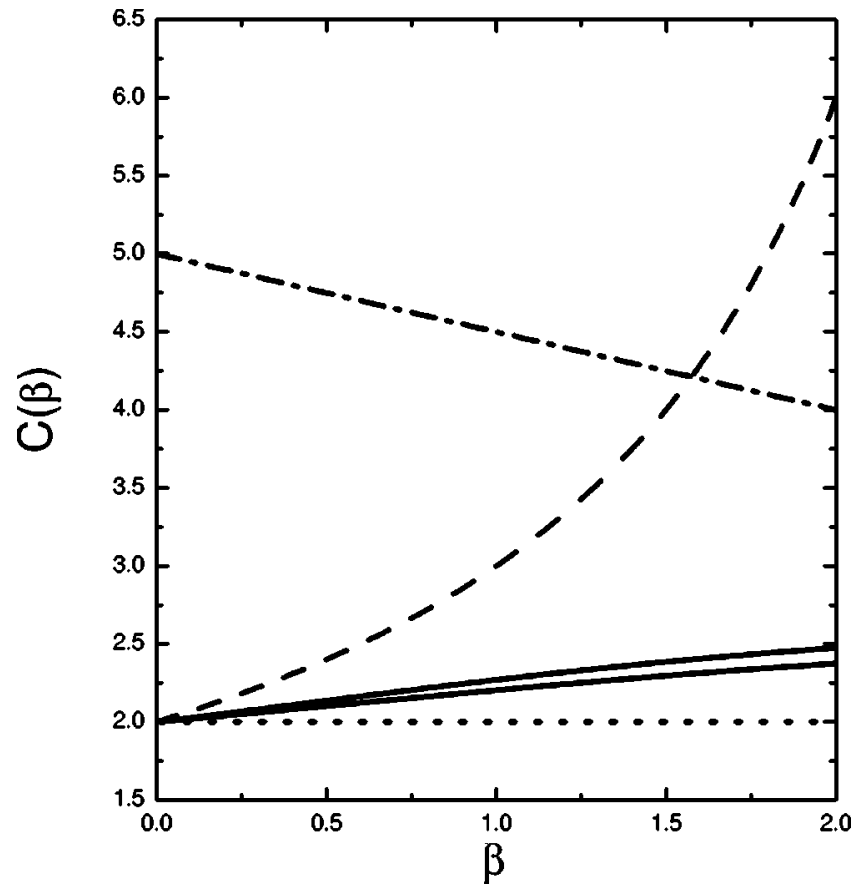

FIG. 3. Bounds for the average residence time for $d=1$ with $n=2$. Here $C_{-}(\beta) \leqslant(2-\beta)\left\langle t_{r}\right\rangle \leqslant C_{+}(\beta)$. The ascending dashed curve is the upper bound given by Eq. (33) and the descending line is the upper bound given by Eq. (32). The horizontal line is the lower bound $C_{-}=2$ common to both equations. Solid lines are the tighter $C_{ \pm}$bounds obtained in the Appendix.

The average over the disorder is calculated using Eq. (27):

$$
\left\langle e^{\beta \Delta}\right\rangle=\int_{0}^{\infty} d \Delta P_{1}(\Delta) e^{\beta \Delta}=2 \int_{0}^{\infty} d \Delta e^{-\Delta(2-\beta)}=\frac{2}{2-\beta},
$$

for $\beta<\beta_{c}=2$.

Thus, the average residence time is bounded as

$$
2 \leqslant(2-\beta)\left\langle t_{r}\right\rangle \leqslant 5-\frac{\beta}{2}
$$

and diverges when $\beta \rightarrow \beta_{c}=2$. These bounds are shown in Fig. 3.

Above the critical temperature, the walker falls into traps (two-cycles) which have finite average residence time. This allows normal diffusion for long time scales. However, the walker eventually falls into a sink which is analogous to an absorbing state. Notice that in general, sinks are not couples. Sinks are absolute traps arising from the finite connectivity $n$. Couples are dynamical traps and temperature allows the escaping from couples but not from sinks. Below the critical temperature $T_{1}=1 / \beta_{1}=1 / 2$, the system falls into an out-ofequilibrium "glassy" phase where the walker explores deeper and deeper (more stable) couples and presents aging phenomena such as in Bouchaud's trap model [10]. This scenario has been called weak ergodicity breaking by Bouchaud and co-workers [13]. 


\section{B. Improved bounds for the residence time}

The previous simple calculations give insight on the physical mechanism involved in the divergence of the residence time, but we can obtain better estimates for the bounds on the residence time, which work for general values of $n$. Using the ordered set of distances, we have that $D_{j} \leqslant D_{a(b) j}$ $\leqslant D_{j}+D_{0}$. The second inequality expresses the fact that our definition of the set of values $D_{j}$ implies that a $d$ sphere of radius $D_{j}+D_{0}$, centered at point $a$ (or $b$ ) contains at least $j$ points. Thus, we can bound the normalization factors in Eq. (28) as follows: $Z_{2} \leqslant Z_{a(b)} \leqslant Z_{1}$, where $Z_{1}=e^{-\beta D_{0}}$ $+\sum_{j=1}^{n-1} e^{-\beta D_{j}}$ and $Z_{2}=e^{-\beta D_{0}}\left[1+\sum_{j=1}^{n-1} e^{-\beta D_{j}}\right]$. This implies that $p_{1} \leqslant p_{a(b)} \leqslant p_{2}$, with $p_{1}=e^{-\beta D_{0} / Z_{1}}$ and $p_{2}$ $=e^{-\beta D_{0} / Z_{2}}$, which allows us to bound the residence time: $t_{r_{1}} \leqslant t_{r} \leqslant t_{r_{2}}$, with $t_{r_{1}}=p_{1} /\left(1-p_{1}\right)$ and $t_{r_{2}}=p_{2} /\left(1-p_{2}\right)$.

To average over the disorder, one needs the joint PDF of the distances of the nearest neighbors to the couple [Eq. (23)]. For $d=1$ one obtains, again for $\beta<\beta_{c}=2$,

$$
2 \leqslant(2-\beta)\left\langle t_{r}\right\rangle \leqslant \frac{6}{3-\beta} \text { for } n=2
$$

and

$$
I_{\beta}(n) \leqslant(2-\beta)\left\langle t_{r}\right\rangle \leqslant \frac{3 I_{\beta}(n)}{3-\beta} \text { for } n>2 \text {, }
$$

with

$$
I_{\beta}(n)=2 \int_{0}^{\infty} \frac{\prod_{i=1}^{n-2}\left(d x_{i} e^{-x_{i}}\right)}{1+\sum_{j=1}^{n-2} \prod_{i=1}^{j} e^{-\beta x_{i} / 2}}=\int_{0}^{1} \frac{\prod_{i=1}^{n-2} d y_{i}}{1+\sum_{j=1}^{n-2} \prod_{i=1}^{j} y_{i}^{\beta / 2}}
$$

In Fig. 3, we give an example of the relationship between these bounds and the exact value of $\left\langle t_{r}\right\rangle$, for the case $n$ $=2$. In fact, the comparison is made not with the exact value but with very tight bounds to it (solid lines in Fig. 3) that have no simple analytical form and have only been obtained for the particular case of $d=1$ and $n=2$ (see the Appendix).

It is easy to see that $2 /(n-1)<I_{\beta}(n)<2$ for all values of $\beta$. Thus, by looking at the bounds we can deduce that $\left\langle t_{r}\right\rangle$ too diverges when $\beta \rightarrow \beta_{c}=2$, for all finite values of $n$.

The function $I_{2}(n)$ can be proven to be a decreasing function of $n$. But it would be interesting to know whether it tends to a positive constant as $n \rightarrow \infty$, or it tends to zero. We have not been able to calculate this limit analytically. Nevertheless, numerical results (see Fig. 4) show that $I_{2}(n)$ seems to decrease toward a positive constant $(\sim 0.56)$. This suggests that a glass transition also exists in the limit $n$ $\rightarrow \infty$.

Moreover, as $n$ grows, the decay of $I_{2}(n)$ is very slow compared to the exponential decrease of density of sinks $\left[C(n) \sim 4 / 3^{n}\right]$. Thus, the divergence of the residence time should not depend on couples inside the sinks; it seems to be

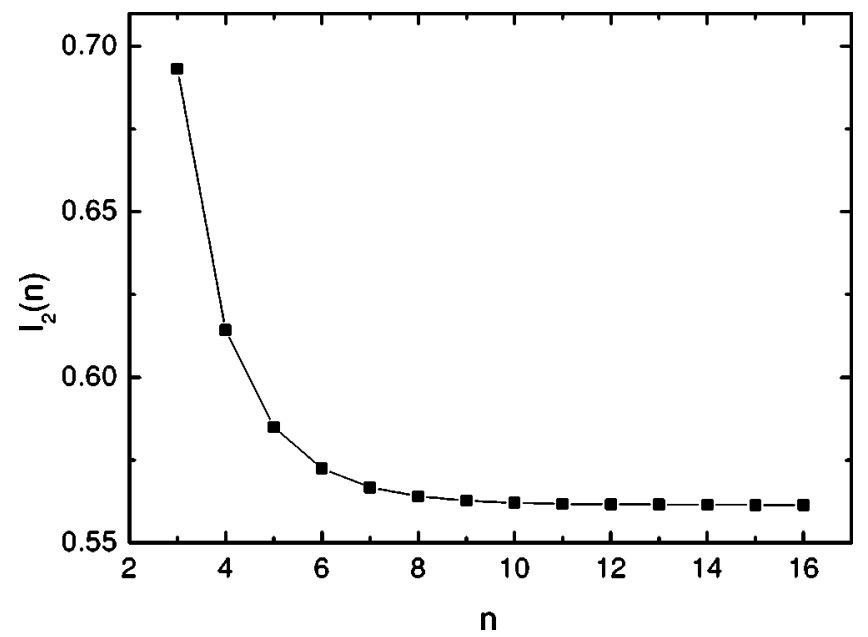

FIG. 4. Numerical calculation of $I_{2}(n)$ as a function of $n$ showing that $I_{2}(n)$ tends to a positive constant.

produced only by couples in the GSCC. This suggests that the glass transition occurs to walkers in the GSCC before they fall into sinks.

\section{Distribution of residence times}

The origin of the glass transition that appears in $d=1$ is related to the trapping times $\operatorname{PDF} P\left(t_{r}\right)$. Exact analytical results are difficult to obtain. Nevertheless, the probability distribution for the approximate residence times $t_{r_{1}}$ and $t_{r_{2}}$ (defined in the preceding section) gives an idea of its behavior. For the case $n=2$, we obtain

$$
\begin{aligned}
P_{1}\left(t_{r_{1}}\right) & =\int_{0}^{\infty} d D_{0} d D_{1} P_{d}\left(D_{0}, D_{1}\right) \delta\left[t_{r_{1}}-e^{-\beta\left(D_{0}-D_{1}\right)}\right] \\
& =\frac{2}{\beta} t_{r_{1}}^{-(2 / \beta+1)} \\
P_{2}\left(t_{r_{2}}\right) & =\int_{0}^{\infty} d D_{0} d D_{1} P\left(D_{0}, D_{1}\right) \delta\left[t_{r_{2}}-e^{\beta\left(D_{1}\right)}\right] \\
& =\frac{6}{\beta} t_{r_{2}}^{-(2 / \beta+1)}\left[1-\frac{1}{t_{r_{2}}^{\beta}}\right]
\end{aligned}
$$

The divergence of the average residence time is a direct consequence of the fact that the tail of these distributions decays with exponent $\gamma<2$ when $\beta>2$. Thus, the behavior of the walker is dominated by couples which have far apart nearest neighbors.

\section{GENERALIZATION TO HIGHER DIMENSIONS AND DIFFERENT COST FUNCTIONS}

For higher-dimensional systems $(d>1)$, the calculations of the properties of the system become harder but some estimates can be obtained. 


\section{A. Asymptotic behavior of the residence times}

It is possible to calculate the behavior of $\left\langle t_{r_{1}}\right\rangle$ and $\left\langle t_{r_{2}}\right\rangle$ when $n=2$. For $d \geqslant 2$ and $\beta \gg 1$, the calculated asymptotic bounds to $\left\langle t_{r}\right\rangle$ are

$$
\frac{1}{\left[d+B\left(\frac{d+1}{2}, \frac{1}{2}\right)\right]^{d}} \leqslant \frac{\left\langle t_{r}\right\rangle}{C_{d} \exp \left[E_{d} \beta^{d /(d-1)}\right]} \leqslant 1
$$

with

$$
\begin{gathered}
E_{d}=(d-1)\left(d A_{d}^{1 / d}\right)^{-d /(d-1)}, \\
C_{d}=\sqrt{\frac{2}{d-1} B\left(\frac{d+1}{2}, \frac{1}{2}\right)^{d} \Gamma\left(\frac{d}{2}+1\right)^{(3-2 d) /(2 d-2)}} \\
\times d ! d^{\left(2 d^{2}-3\right) /(2 d-2)} \pi^{\left(d^{2}-d / 2-1\right) /(2 d-2)}
\end{gathered}
$$

where $A_{d}$ is given by Eq. (13).

Thus, there is no glass transition when $d \geqslant 2$ for the linear cost function $E\left(D_{i j}\right)=D_{i j}$. However, as we show below, there is a specific cost function which yields a glass transition for each dimension.

\section{B. Generalized cost functions}

Consider the general case where the transition rates have the form $W_{i \rightarrow j}=\exp \left[-\beta E\left(D_{i j}\right)\right] / Z_{i}$ with an arbitrary cost function $E\left(D_{i j}\right)$. In the symmetric approximation, the average residence time is

$$
\left\langle t_{r s}\right\rangle=\int_{0}^{\infty} d D_{1} d D_{0} P_{d}\left(D_{1}, D_{0}\right) e^{\beta\left[E\left(D_{1}\right)-E\left(D_{0}\right)\right]},
$$

where $P_{d}\left(D_{1}, D_{0}\right)$ is given by Eq. (24) (using $k=1$ ). It is clear that the two competing factors which create the possibility of a glass transition are (i) the tail of $P_{d}\left(D_{1}, D_{0}\right)$ and (ii) the exploding $\exp \left[\beta E\left(D_{1}\right)\right]$ factor. As it can be seen from Eq. (23), the tail of $P_{d}\left(D_{1}, D_{0}\right)$ is $P_{d}\left(D_{1}, D_{0}\right)$ $\propto \exp \left(-A_{d} D_{1}^{d}\right)$, where $A_{d}$ is given by Eq. (13). Thus, the symmetric approximation leads to

$$
\left\langle t_{r s}\right\rangle \propto \int_{D_{0}}^{\infty} d D_{1} e^{\beta E\left(D_{1}\right)-A_{d} D_{1}^{d}} .
$$

Consider, for instance, the family of cost functions: $E$ $=D_{i j}^{\alpha}$. If $\alpha$ is smaller than $d$, the residence time is always finite and no glassy behavior is observed (as we have previously observed in the case $d=2$ and $\alpha=1$ ). The limiting case $T=1 / \beta \rightarrow 0$ will be examined elsewhere. On the other hand, if $\alpha>d$, the system is always in the glassy state for any value of $\beta$.

If the cost function exponent is equal to the space dimensionality, $\alpha=d$, the two terms in the argument of exponential function in Eq. (42) can compete independently of the value of $D_{1}$ and a glass transition occurs at $\beta_{d}=A_{d}=\pi^{d / 2} / \Gamma(d / 2$ +1 ). For example, $\beta_{1}=2$ for $d=1$ (as seen previously), $\beta_{2}=\pi$ for $d=2$, and $\beta_{3}=4 \pi / 3$ for $d=3$.
This kind of glass transition generalizes those found in trap models. In trap models, once the tail of the barrier distribution is given (say, a Gaussian tail as in Ref. [14]), one cannot carpenter the Arrhenius term to compete with it. This is different in our exploratory random walk model. In our case what is given is not the barrier distribution $P(E)$, but the distance distribution $P\left(D_{i j}\right)$ which characterizes the landscape. Since the transition probability for exploratory random walks depends on an arbitrary cost function $E\left(D_{i j}\right)$, one can always find what condition must be satisfied by the cost function so that a glass transition occurs.

\section{Lévy flights}

From Eq. (42), one sees that any $\alpha<d$ prevents the glass transition. In particular, one can study the limit $\alpha \rightarrow 0$, i.e., $E\left(D_{i j}\right)=\ln D_{i j}$. In this case, a hopping process with the power-law tail, $W_{i \rightarrow j}=D_{i j}^{-\beta} / \Sigma_{j \in \mathrm{NN}} D_{i j}^{-\beta}$ is found. Transition probabilities with power-law ("Lévy") tails lead to finite average residence times for any value of $d$. Such Lévy exploration process has been considered, for instance, in Refs. [27-29].

But glass transitions may occur if the distribution of point distances also has power-law tails. For example, suppose that the points coordinates are drawn from some distribution that produces $P\left(D_{1}, D_{0}\right) \propto D_{1}^{-b}$. Then, the diverging part of the residence time integral has the form $\left\langle t_{r s}\right\rangle \propto \int d D_{1} D_{1}^{\beta-b}$. This means that a glass transition occurs if $\beta \geqslant \beta_{c}=b-1$.

\section{CONCLUSION}

We have introduced a random walk in a disordered medium dependent on a control parameter which tunes the system from an ergodic to a nonergodic regime through a glass transition. The random walk is similar to a hopping process in a multivalley landscape. The $d=1$ "local minima" or traps emerge as two-cycle attractors produced by sites which are mutual nearest neighbors. For $d=1$ these traps present a power-law distribution of trapping times and the average trapping time diverges for $T \leqslant T_{c}=1 / 2$, leading to a "glass" transition in the weak ergodicity breaking scenario of Bouchaud's trap model $[10,12]$. Given any distribution of distances and any dimensionality, one can always find a cost function for which a similar glass transition occurs.

The analytical treatment to estimate the bounds of the average residence time has been possible because we have considered finite connectivity. The finite value of $n$ is also responsible for the appearance of special sets of points (sinks and sources) in the directed graph where the walks are performed. Sinks are absolute, inescapable topological traps insensitive to temperature. Couples (that produce two-cycles) are relative traps that can be escaped with finite temperature. We have considered a possible glass transition involving couples before the walker falls into sinks. Given the behavior of the bounds to the average residence time, we also expect a glass transition to appear in the fully connected network with $n=N-1$, where no sinks exist, only couples.

Concerning the application of our model to exploratory behavior, one (a posteriori) obvious conclusion is that free 
exploration (diffusion or superdiffusion) occurs when the tail of the hop's PDF decays slower than the tail of the first neighbors distances' PDF. However, if costs are associated with the traveling distance, the marginal scenario, where the two PDF tails $(\alpha=d)$ compete, may be of interest. We conjecture that working at the glass transition border may be optimal when high costs are associated with travel distances (this will be presented elsewhere).

The two-cycle phase (at zero temperature) and the glassy behavior of the stochastic tourist walk may be compared to similar behaviors in neural and Kauffman networks. Such comparison is not possible by using the random map. The random walk in a disordered graph introduced here is interesting because it generalizes the usual trap models: in the tourist walk, traps are made of dynamical cycles instead of energy minima. We are currently studying the stochastic tourist walk with memory $\tau>0$, so that traps are cycles with large periods (similar to what happens in Kauffmann networks and asymmetric neural networks [17]). We expect that the relevant features should be the same as those observed in the present work. In short, if one wants to escape from vicious cycles, small perturbations may not be sufficient: freedom only appears above a finite temperature $T_{c}$.

\section{ACKNOWLEDGMENTS}

The authors acknowledge useful conversations with $\mathrm{J}$. Arenzon, N. Caticha, R. Dickman, J. R. Drugowich de Felcio, J. F. Fontanari, M. A. Idiart, G. F. Lima, D. A. Stariolo, A. C. Roque da Silva, R. da Silva, and C. Yokoi. O.K. has been supported by FAPESP.

\section{APPENDIX: IMPROVED BOUNDS}

In this appendix, we derive tighter bounds than those given by Eqs. (32) and (33), but they are only valid in the special case of $d=1$ and $n=2$.

Consider first a couple of points, $a$ and $b$, separated by a distance $D_{0}$. To calculate $Z_{a}$ and $Z_{b}$ [see Eq. (28)], one only needs the positions of $a$ and $b$ and their two nearest neighbors. The first neighbor to each of them is, by definition, the other member of the couple. Since the distribution of the distances of the neighbors to the couple is known, one needs to determine which of these neighbors is the second-nearest neighbor to $a$ and which is the SNN to $b$.

Without loss of generality, we assume $b$ to be the rightmost point of the couple. The first neighbor to the couple (called FNNC) is taken to be placed at a distance $D_{1}$ (with $D_{1}>D_{0}$ ), and at the right of $b$, which makes it the SNN to $b$. Now the task is reduced to the determination of the SNN to point $a$.

When one considers the position of the second-nearest neighbor to the couple (called SNNC), at a distance $D_{2}$ (with $D_{2}>D_{1}$ ), three possibilities arise for the SNN of $a$.

(I) $D_{2}>D_{1}+D_{0}$. In this case, the SNN to $a$ is the FNNC, and its distance to point $a$ is $D_{1}+D_{0}$.

(II) $D_{2}<D_{1}+D_{0}$, but it is placed to the left of $a$. In this case, the SNN to $a$ is the SNNC, and its distance to point $a$ is $D_{2}$.

(III) $D_{2}<D_{1}+D_{0}$, but it is placed to the right of $b$. In this case, one needs to know the position of further neighbors to the couple to obtain the SNN to $a$. If they are placed at distances larger than $D_{1}+D_{0}$, then the SNN to $a$ would be the SNNC. Otherwise, one would have to know which neighbors, of those having distances smaller than $D_{1}+D_{0}$, are placed to the left of $a$.

The corresponding residence times can be calculated exactly for cases (I) and (II).

For case (I), the partition functions are $Z_{a \mathrm{I}}(\beta)=e^{-\beta D_{0}}$ $+e^{-\beta\left(D_{0}+D_{1}\right)}$ and $Z_{b \mathrm{I}}(\beta)=e^{-\beta D_{0}+e^{-\beta D_{1}}}$. For case (II), they are $Z_{a \mathrm{II}}(\beta)=e^{-\beta D_{0}}+e^{-\beta D_{2}}$ and $Z_{b \mathrm{II}}(\beta)=e^{-\beta D_{0}}$ $+e^{-\beta D_{1}}$.

Equations (29) and (28) yield the residence times

$$
\begin{gathered}
t_{r \mathrm{I}}(\beta)=\frac{\left(1+e^{\left.-\beta D_{0}\right) / 2+2 e^{\beta\left(D_{1}-D_{0}\right)}}\right.}{1+e^{-\beta D_{0}+e^{-\beta D_{1}}}}, \\
t_{r \mathrm{II}}(\beta)=\frac{2 e^{-\beta D_{0}}+\left(e^{-\beta D_{1}}+e^{-\beta D_{2}}\right) / 2}{e^{-\beta D_{1}}+e^{-\beta D_{2}}+e^{\beta\left(D_{0}-D_{1}-D_{2}\right)}} .
\end{gathered}
$$

The problem with $t_{r \text { III }}$ is that to calculate it exactly, one needs to break case (III) into an infinity of particular cases. For our purposes, it suffices to calculate some bounds to its real value. As it has been discussed in case (III), the distance of point $a$ to its SNN satisfies $D_{2}<D_{S N N a}<D_{0}+D_{1}$. Therefore, it is clear that the corresponding residence time must satisfy $t_{r \mathrm{I}}<t_{r \mathrm{III}}<t_{r \mathrm{II}}$.

Remembering that the probability for a given point to be at one side of the couple is $1 / 2$, and averaging, one obtains the bound

$$
\begin{aligned}
\int & d D_{0} d D_{1} d D_{2} P_{1}\left(D_{0}, D_{1}, D_{2}\right)\left[t_{r \mathrm{I}} \Theta\left(D_{2}-D_{1}-D_{0}\right)\right. \\
& \left.+\frac{t_{r \mathrm{I}}+t_{r \mathrm{II}}}{2} \Theta\left(D_{1}+D_{0}-D_{2}\right)\right] \leqslant\left\langle t_{r}\right\rangle \\
\leqslant & \int d D_{0} d D_{1} d D_{2} P_{1}\left(D_{0}, D_{1}, D_{2}\right)\left[t_{r \mathrm{I}} \Theta\left(D_{2}-D_{1}-D_{0}\right)\right. \\
& \left.+t_{r \mathrm{II}} \Theta\left(D_{1}+D_{0}-D_{2}\right)\right] .
\end{aligned}
$$

We have calculated these integrals numerically, and the resulting bounds are presented as full lines in Fig. 3.
[1] D.S. Fisher, Phys. Rev. A 30, 960 (1984).

[2] G.T. Barkema, P. Biswas, and H. van Beijeren, Phys. Rev. Lett. 87, 170601 (2001).

[3] H. Freund and P. Grassberger, Physica A 190, 218 (1992).
[4] D. Gale, J. Propp, S. Sutherland, and S. Troubetzkoy, Math. Intell. 17, 48 (1995).

[5] G.F. Lima, A.S. Martinez, and O. Kinouchi, Phys. Rev. Lett. 87, 010603 (2001). 
[6] H.E. Stanley and S.V. Buldyrev, Nature (London) 413, 373 (2001).

[7] O. Kinouchi, A.S. Martinez, G.F. Lima, G.M. Lourenço, and S. Risau-Gusman, Physica A 315, 665 (2002).

[8] Y. Limoge and J.L. Bocquet, Phys. Rev. Lett. 65, 60 (1990).

[9] J.C. Dyre, Phys. Rev. Lett. 58, 792 (1987).

[10] J.-P. Bouchaud, J. Phys. I 2, 1705 (1992).

[11] J.C. Dyre, Phys. Rev. B 51, 12276 (1995).

[12] C. Monthus and J.-P. Bouchaud, J. Phys. A 29, 3847 (1996).

[13] B. Rinn, P. Maass, and J.-P. Bouchaud, Phys. Rev. B 64, 104417 (2001).

[14] R.A. Denny, D.R. Reichman, and J.-P. Bouchaud, e-print cond-mat/0209020.

[15] W.A. Little and G.L. Shaw, Math. Biosci. 39, 281 (1978).

[16] J.F. Fontanari and R. Koberle, Phys. Rev. A 36, 2475 (1987).

[17] U. Bastolla and G. Parisi, J. Phys. A 31, 4583 (1998).

[18] U. Bastolla and G. Parisi, Physica D 98, 1 (1996).

[19] B. Derrida and H. Flyvbjerg, J. Phys. (Paris) 48, 971 (1987).

[20] W. Feller, An Introduction to Probability Theory and its Appli- cations (Wiley, New York, 1968), Vols. 1 and 2.

[21] S.N. Dorogovtsev, J.F.F. Mendes, and A.N. Samukhin, Phys. Rev. E 64, 025101(R) (2001).

[22] M.E.J. Newman, S.H. Strogatz, and D.J. Watts, Phys. Rev. E 64, 026118 (2001).

[23] O. Kinouchi, A.S. Martinez, G.F. Lima, and M.A. Idiart (unpublished).

[24] T.F. Cox, Biometrics 37, 367 (1981).

[25] Handbook of Mathematical Functions, edited by M. Abramowitz and I.A. Stegun (Dover, New York, 1972).

[26] A.G. Percus and O.C. Martin, J. Stat. Phys. 94, 739 (1999).

[27] G.M. Viswanathan, V. Afanasyev, S. Buldyrev, E.J. Murphy, P.A. Prince, and H.E. Stanley, Nature (London) 381, 413 (1996).

[28] E. Barkay and V.N. Fleurov, Phys. Rev. E 58, 1296 (1998).

[29] G.M. Viswanathan, S. Buldyrev, S. Havlin, M.G.E. da Luz, E.P. Raposol, and H.E. Stanley, Nature (London) 401, 911 (1999). 